\title{
Correction to: Integrating Caries Detection Devices into Clinical Practice
}

\author{
Stephen Abrams
}

\section{Correction to:}

Chapter 24 in: A. Ferreira Zandona, C. Longbottom (eds.),

Detection and Assessment of Dental Caries, https://doi.org/10.1007/978-3-030-16967-1_24

Dr. Stephen Abrams name had been misspelt in the original version of the book in chapter 24. The author's name has now been corrected. 